\title{
1 Sampling principles for biodiversity study
}

2 Xubin Pan

3 Institute of Plant Quarantine, Chinese Academy of Inspection and Quarantine, Beijing 100029,

4 China

$5 \quad$ E-mail: xubin.hu.pan@gmail.com

6

\section{Abstract}

8 Sampling is a fundamental tool in ecology and critical for biodiversity measurement. However,

9 basic principles of biodiversity sampling have been overlooked for many years. In this paper, I

10 proposed and explored five principles of sampling for a specific area and biodiversity study.

11 The first principle of sampling, species increasing with area, is that the number of species

12 increases with the area. The second principle of sampling, individuals increasing with area, is

13 that the number of individuals increases with the area. The third principle of sampling, sum of

14 species number, is that the sum of species number in one area and species number in another

15 area is no less than the total species number in the two areas. The fourth principle of sampling,

16 individual complement, is that the sum of the mathematical expectation of individual number

17 of one or several species in the area $a$ and that of the same one or several species in the area

$18 A-a$ is the total individual number $N$ of the same one or several species in the total area $A$. The

19 fifth principle of sampling, species-area theory, is that the sum of the mathematical expectation

20 of number of species in the area $a$ and that of number of species lost if area $A-a$ is cleared is the

21 total species number $M$ in the total area $A$.

22 Keywords: Individuals-area relationship, Species-area relationship, Endemics-area relationship 


\section{Introduction}

Sampling is a fundamental tool in ecology. Because the complete investigation of one ecological question maybe involves a large area and long time, it will cost too much time and money, and leads to infeasible in practice. Thus, sampling is unavoidable in the ecology study. However, due to the complexity of ecosystem, such as interactions among organisms and environment and their large spatial-temporal heterogeneity, how to sampling should be very carefully, which will decide how to analyze the data from the sampling and the credibility of the results. Thus, solid sampling theory is very important for ecology, although it didn't get too much concern recently. As the core of the ecology, the spatial relationship between the species and the area can be a good start for rethink the sampling theory.

Individual-area relationship (IAR), species-area relationship (SAR) and endemics-area relationship (EAR) are important concepts in biodiversity conservation and habitat preservation (WCMC, 1992; Kinzig and Harte, 2000; Connor et al., 2000; Green and Ostling, 2003; Chris et al., 2004; World Resources Institute, 2005). However, there is still much debating over the estimation of extinction rate based on SAR, which tends to make overestimation when compared with observed extinction (Pimm and Askins, 1995; Rosenzweig, 1995; Harte and Kinzig, 1997; He and Hubbell, 2011; Pan, 2013). One explanation is that such estimation includes certain species that are "committed to extinction" instead of going extinct right away after habitat clearing (Heywood, 1994; Tilman, 1994; Mace et al., 2003). Recently, He and Hubbell (2011) suggested that the reason was the difference of between sampling areas based SAR and EAR, because a sample area of a species for extinction is often larger than a sample 
45 area of the same species for existence. Among these controversies, the core problem is the

46 sampling of biodiversity measurement, the basic principles of which have been overlooked for

47 many years.

48

49 However, much work has been focused on statistical or mathematical calculation based on

50 SAR, rather than the biological and ecological implication, especially the basic principles for

51 biodiversity sampling (Turner and Tjørve, 2005). Are there common laws for sampling in

52 biodiversity measurement? Here, we proposed five sampling principles for biodiversity study

53 in a specific area and the last two were proved, focusing on the change of species number and

54 individuals for one or several species with a changing area. This analysis will be helpful for the

55 establishment of theory platform for biodiversity and other ecological discussion.

56

57 Theoretical Frame

58 Biodiversity not just study the number of species, but also the amount of individuals. For the

59 sampling problems of biodiversity, is the relationship between the area and the number of

60 species and amount of individuals. Usually, it is thought the relationship between the number

61 of species and the area is saturation curve, and the relationship between the amount of

62 individuals and the area is increasing curve. The shape of this curve is influenced by the spatial

63 distribution and sampling collection/statistic.

64

65 Sampling principles for biodiversity measurement

66 The first principle of sampling, species increasing with area, is that the number of species 
67 increases with the area. Assume the number of species is $m$ in the area $a$, when the sampling

68 area increases from $a$ to $a^{\prime}$, the number of species in area $a^{\prime}$ is $m^{\prime}$, and $m^{\prime} \geq m$. If no new

69 species emerges in the area $a^{\prime}-a, m^{\prime}=m$.

70

71 The second principle of sampling, individuals increasing with area, is that the number of

72 individuals increases with the area. Assume the number of individuals is $n$ in the area $a$,

73 when the sampling area increases from $a$ to $a^{\prime}$, the number of individuals in area $a^{\prime}$ is $m^{\prime}$,

74 and $n^{\prime} \geq n$. If there no new individuals emerges in the area $a^{\prime}-a, n^{\prime}=n$.

75

76

77

78

79

80

81

82

83

84

85

86

87 The fifth principle of sampling, species-area theory, is that the sum of the mathematical

88

The third principle of sampling, sum of species number, is that the sum of species number in one area and species number in another area is no less than the total species number in the two areas. Assume the number of species is $m_{1}$ in the area $a_{1}$, the number of species is $m_{2}$ in the area $a_{2}$, the number of species in area $a_{1}+a_{2}$ is $m^{\prime}$, and $m^{\prime} \leq m_{1}+m_{2}$. If there is no overlapping species between areas $a_{1}$ and $a_{2}, m^{\prime}=m_{1}+m_{2}$.
The fourth principle of sampling, individual complement, is that the sum of the mathematical expectation of individual number of one or several species in area $a$ and that of individual number of the same one or several species in area $A-a$ is the total individual number $N$ of the same one or several species in the total area $A$.

expectation of number of species in area $a$ and that of number of species lost if area $A-a$ is 
89 cleared is the total species number $M$ in the total area $A$.

90

91 Mathematical proof for the fourth and fifth principles of biodiversity sampling

92 While the first, second and third principles of biodiversity sampling are straightforward and

93

94

95

96

97

98

99

100 $\operatorname{ME}\left(I_{a}\right)=\sum_{i=1}^{k} I_{a, i} P_{i}$

The individual-area relationship (IAR) $I_{a}$ in area $a$ and $I_{A-a}$ in area $A$ - $a$, for one sampling, if the sampling area is $a$ and the number of individuals in this area is $n$, there is always one-one corresponding area of $N-n$ individuals of the species in area $A-a$ ( $A$ is the total area, and $N$ is the total individuals for one or several species in area $A$ ).

The mathematical expectation of individual-area relationship in area $a$ is where $M E\left(I_{a}\right)$ are the expected individuals for the individuals-area relationship in area $a$ in

102 the $i$ th sampling, $k$ is the total samples, and the $P_{i}$ is the possibility of the corresponding $i$ th 103 sampling.

104 The mathematical expectation of individual-area relationship in area $A-a$ is $M E\left(I_{A-a}\right)=\sum_{i=1}^{k} I_{A-a, i} Q_{i}$

105 where $M E\left(I_{A-a}\right)$ are the expected individuals for the individual-area relationship in area $A-a$ 106 in the $i$ th sampling, and the $Q_{i}$ is the possibility of the corresponding $i$ th sampling.

107 For any specific sampling $i$, there is always a one-one corresponding $I_{a, i}+I_{A-a, i}=N$, and the 
$P_{i}=Q_{i}$ in this situation.

$$
\begin{gathered}
M E\left(I_{a}\right)+M E\left(I_{A-a}\right)=\sum_{i=1}^{k} I_{a, i} P_{i}+\sum_{i=1}^{k} I_{A-a, i} Q_{i}=\sum_{i=1}^{k}\left(I_{a, i}+I_{A-a, i}\right) * P_{i}=\sum_{i=1}^{k} N P_{i}=\mathrm{N} \sum_{i=1}^{k} P_{i} \\
=\mathrm{N}
\end{gathered}
$$

109

110 The number of species in area $a$ is $m$, then $S_{a}=m$, where species-area relationship (SAR) $S_{a}$ is

111 the function of the species number with area $a$. The number of species lost in area $A-a$ if the

112 area $A-a$ is cleared is $M-m$, endemics-area relationship (EAR) $E_{A-a}$ is the number of species

113 disappearing if area $A-a$ is cleared.

114 The mathematical expectation of Species-area relationship in area $a$ is

$M E\left(S_{a}\right)=\sum_{i=1}^{k} S_{a, i} P_{i}$

115 where $S_{a, i}$ is the species number in area $a$ in the $i$ th sampling, $k$ is the total samples, and the $P_{i}$

116 is the possibility of the corresponding $i$ th sampling.

117 The mathematical expectation of Endemics-area relationship in area $A$ - $a$ is

$M E\left(E_{A-a}\right)=\sum_{i=1}^{k} E_{A-a, i} Q_{i}$

118 where $E_{A-a, i}$ is the number of species disappear if area $A$ - $a$ is lost in the $i$ th sampling, and the $Q_{i}$

119 is the possibility of the corresponding $i$ th sampling.

120 For any specific sampling $i$, there is always a one-one corresponding $S_{a, i}+E_{A-a, i}=M$, and

121 the $P_{i}=Q_{i}$ in this situation.

$$
\begin{gathered}
M E\left(S_{a}\right)+M E\left(E_{A-a}\right)=\sum_{i=1}^{k} S_{a, i} P_{i}+\sum_{i=1}^{k} E_{A-a, i} Q_{i}=\sum_{i=1}^{k}\left(S_{a, i}+E_{A-a, i}\right) P_{i} \\
=\sum_{i=1}^{k} M P_{i}=M \sum_{i=1}^{k} P_{i}=M
\end{gathered}
$$




\section{Conclusions}

124 Sampling is a fundamental tool in ecology and critical for biodiversity measurement. However, 125 basic principles of biodiversity sampling have been overlooked for many years. In this paper, I 126 proposed and explored five principles of sampling for a specific area and biodiversity study.

127 The first principle of sampling, species increasing with area, is that the number of species

128 increases with the area. The second principle of sampling, individuals increasing with area, is

129 that the number of individuals increases with the area. The third principle of sampling, sum of

130 species number, is that the sum of species number in one area and species number in another

131 area is no less than the total species number in the two areas. The fourth principle of sampling,

132 individual complement, is that the sum of the mathematical expectation of individual number

133 of one or several species in the area $a$ and that of the same one or several species in the area

$134 A-a$ is the total individual number $N$ of the same one or several species in the total area $A$. The

135 fifth principle of sampling, species-area theory, is that the sum of the mathematical expectation

136 of number of species in the area $a$ and that of number of species lost if area $A-a$ is cleared is the 137 total species number $M$ in the total area $A$.

139 Acknowledgments

140 I warmly thank Fengqiao Liu at Arizona State University and Dr. James Rosindell in 141 University of Leeds for the helpful suggestions for the manuscript revision.

\section{References}


144 Achard F et al. 2002. Determination of deforestation rates of the world's humid tropical forests.

$145 \quad$ Science 297: 999-1002.

146 Connor EF, Courtney AC and Yoder JM. 2000. Individuals-area relationships: the relationship 147 between animal population density and area. Ecology 81: 734-748.

148 Green JL and Ostling A. 2003. Endemics-area relationships: the influence of species 149 dominance and spatial aggregation. Ecology 84: 3090-3097.

150 Green JL, Harte J and Ostling A. 2003. Species richness, endemism, and abundance patterns: 151 tests of two fractal models in a serpentine grassland. Ecology Letters 6: 919-928.

152 Harte J and Kinzig AP. 1997. On the implications of the species-area relationships for 153 endemism, spatial turnover, and food web patterns. Okios 80: 417-427.

154 Harte J, Kinzig AP and Green J. 1999. Self-similarity in the distribution and abundance of $155 \quad$ species. Science 284: 334-336.

$156 \mathrm{He} F$ and Hubbell SP. 2011. Species-area relationships always overestimate extinction rates 157 from habitat loss. Nature 473: 368-371.

158 Heywood VH, Mace GM, May RM and Stuart SN. 1994. Uncertainties in extinction rates. $159 \quad$ Nature 368: 105.

160 Kinzig AP and Harte J. 2000. Implications of endemics-area relationships for estimates of $161 \quad$ species extinctions. Ecology 81: 3305-3311.

162 Mace GM, Gittleman JL and Purvis A. 2003. Preserving the tree of life. Science 300: 163 1707-1709.

164 Pan, XB. 2013. Fundamental equations for species-area theory. Scientific Reports. 2013, 3: 165 1334. 
166 Pimm SL and Askins RA. 1995. Forest losses predict bird extinctions in eastern North America. 167 Proceedings of National Academic of Sciences 92: 9343-9347.

168 Rosenzweig ML. 1995. Species Diversity in Space and Time. Cambridge University Press, 169 Cambridge.

170 Thomas CD. et al. 2004. Extinction risk from climate change. Nature 427: 145-148.

171 Tilman D, May RM, Lehman CL and Nowak MA. 1994. Habitat destruction and the extinction 172 debt. Nature 371: 365-366.

173 Turner WR and Tjørve E. 2005. Scale-dependence in species-area relationships. Ecography 28: $174 \quad 721-730$.

175 WCMC. 1992. Global Biodiversity: Status of the Earth's Living Resources. Chapman \& Hall, $176 \quad$ London.

177 World Resources Institute. 2005. Millennium Ecosystem Assessment. Ecosystems and Human 178 Well-Being: Biodiversity Synthesis.

179

180 


\section{$181 \quad$ Figures Captions}

182 Figure 1. Relationship between the number of species and amount of individuals and the area

183 Figure 2. Individual-area relationship $I_{a}$ in area $a$ and $I_{A-a}$ in area $A-a$.

184 Figure 3. Species-area relationship $S_{a}$ in area $a$ and endemics-area relationship $E_{A-a}$ in area $A-a$. 


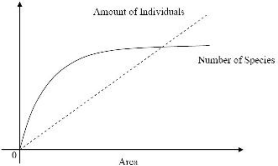




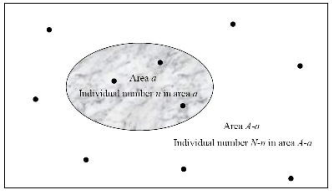




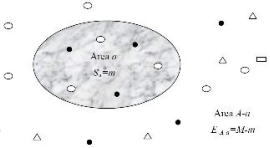

